\title{
A new extension of bivariate FGM copulas
}

\author{
Cécile Amblard ${ }^{1} \&$ Stéphane Girard ${ }^{2}$ \\ ${ }^{1}$ Institut de l'Ingénierie de l'Information de Santé, \\ TIMC - TIMB, Faculté de Médecine, Pav. D, \\ 38706 La Tronche Cedex, France. \\ E-mail : Cecile.Amblard@imag.fr \\ ${ }^{2}$ INRIA Rhône-Alpes, team Mistis, \\ 655, avenue de l'Europe, Montbonnot, \\ 38334 Saint-Ismier Cedex, France. \\ E-mail: Stephane.Girard@inrialpes.fr
}

\begin{abstract}
We propose a new family of copulas generalizing the Farlie-Gumbel-Morgenstern family and generated by two univariate functions. The main feature of this family is to permit the modeling of high positive dependence. In particular, it is established that the range of the Spearman's Rho is $[-3 / 4,1]$ and that the upper tail dependence coefficient can reach any value in $[0,1]$. Necessary and sufficient conditions are given on the generating functions in order to obtain various dependence properties. Some examples of parametric subfamilies are provided.
\end{abstract}

Keywords: Copulas, semiparametric family, measures of association, positive dependence.

AMS Subject classifications: Primary 62H05, secondary 62H20.

\section{Introduction}

A bivariate copula defined on the unit square $I^{2}:=[0,1]^{2}$ is a bivariate cumulative distribution function (cdf) with univariate uniform $I$ margins i.e. verifying the following three properties:

(P1) $C(u, 0)=C(0, v)=0, \forall(u, v) \in I^{2}$,

(P2) $C(u, 1)=u$ and $C(1, v)=v, \forall(u, v) \in I^{2}$,

(P3) $\Delta\left(u_{1}, u_{2}, v_{1}, v_{2}\right):=C\left(u_{2}, v_{2}\right)-C\left(u_{2}, v_{1}\right)-C\left(u_{1}, v_{2}\right)+C\left(u_{1}, v_{1}\right) \geq 0, \forall\left(u_{1}, u_{2}, v_{1}, v_{2}\right) \in I^{4}$, such that $u_{1} \leq u_{2}$ and $v_{1} \leq v_{2}$.

Sklar's Theorem [29] states that any bivariate distribution with cdf $H$ and marginal cdf $F$ and $G$ can be written $H(x, y)=C(F(x), G(y))$, where $C$ is a copula. This result justifies the use of copulas for building bivariate distributions.

One of the most popular parametric family of copulas is the Farlie-Gumbel-Morgenstern (FGM) family defined when $\theta \in[-1,1]$ by

$$
C_{\theta}^{\mathrm{FGM}}(u, v)=u v+\theta u(1-u) v(1-v),
$$

and studied in [7, 10, 18. A well-known limitation to this family is that it does not allow the modeling of large dependences since Spearman's Rho is limited to $\rho \in[-1 / 3,1 / 3]$. Basing on this remark, more general copulas have been introduced in 1960 by Sarmanov [26],

$$
C_{\theta, \phi, \psi}^{\text {Sarmanov }}(u, v)=u v+\theta \phi(u) \psi(v),
$$


an re-discovered in 2004 by Rodríguez-Lallena and Úbeda-Flores [25]. See [14 for an extension of this model. Properties of Sarmanov copulas are studied in [16, 27]. Unfortunately, characterization of admissible parameters $\theta$ and functions $\phi$ and $\psi$ is not tractable to obtain closed-form bounds on the corresponding Spearman's Rho. Thus, several parametric sub-families of (1.2) were introduced. In [21], it is remarked that copulas with quadratic sections [23] are not able to model large dependences. Copulas with cubic sections are thus introduced, with the conclusion that copulas with higher order polynomial sections would increase the dependence degrees but simultaneously the complexity of the model. In [11, two kernel extensions of FGM copulas are studied

$$
C_{\theta, \gamma}^{\mathrm{HK} 1}(u, v)=u v+\theta u(1-u)^{\gamma} v(1-v)^{\gamma}
$$

for $\gamma \geq 1$ and

$$
C_{\theta, \gamma}^{\mathrm{HK} 2}(u, v)=u v+\theta u\left(1-u^{\gamma}\right) v\left(1-v^{\gamma}\right),
$$

for $\gamma \geq 1 / 2$. It is shown that Spearman's Rho can be increased up to approximatively 0.39 while the lower bound remains $-1 / 3$. Another similar extension is

$$
C_{\theta, p, q}^{\mathrm{LX}}(u, v)=u v+\theta u^{p}(1-u)^{q} v^{p}(1-v)^{q},
$$

see [15]. Copulas (1.3) and (1.4) are particular cases of Bairamov-Kotz family [3] defined by

$$
C_{\theta, p, q, n}^{\mathrm{BK}}(u, v)=u^{p} v^{p}\left[1+\theta\left(1-u^{q}\right)^{n}\left(1-v^{q}\right)^{n}\right],
$$

and with associated Spearman's Rho $\rho \in[-0.48,0.501594]$. Moreover, it has been remarked in 12 that dependence degrees arbitrarily close to \pm 1 cannot be obtained with polynomial functions of fixed degree. An alternative approach to generalize the FGM family of copulas is to consider the semi-parametric family of symmetric copulas defined by

$$
C_{\theta, \phi}^{\mathrm{SP}}(u, v)=u v+\theta \phi(u) \phi(v),
$$

with $\theta \in[-1,1]$. It was first introduced in [24, and extensively studied in [1, 2]. Let us precise that, in this paper and in accordance with [22], page 38, a copula $C$ is said to be symmetric if $C(u, v)=C(v, u)$ for all $(u, v) \in I^{2}$. Clearly, this family also includes the FGM copulas (1.1) (which contains all copulas with both horizontal and vertical quadratic sections [23]), the parametric family of symmetric copulas with cubic sections proposed in [21], equation (4.4), and kernel families (1.3), (1.4) and (1.5). It can be shown that, for a properly chosen function $\phi$, the range of Spearman's Rho is extended to $[-3 / 4,3 / 4]$, whereas the upper tail dependence coefficient is always null. We refer to [8] for a very interesting method for constructing admissible functions $\phi$.

In this paper, we propose an extension of the $C_{\theta, \phi}^{\mathrm{SP}}$ family where $\theta$ is a univariate function. This modification allows the introduction of a singular component concentrated on the diagonal $v=u$. Consequently, the modeling of strong positive dependences is possible since this new family can take into account the extremal case of positive functional dependence between two random variables. Moreover, arbitrary upper tail dependence coefficients can be reached in $[0,1]$. The new family is described in Section 2 and the associated Spearman's Rho and tail dependence coefficients are studied in Section 3. Section 4 is dedicated to the dependence properties of this new family of copulas. Finally, some examples of copulas taken in this family are provided in Section 5. Lemmas are postponed to the appendix.

\section{Definition and basic properties}

We consider the family of functions defined on $I^{2}$ by:

$$
C_{\theta, \phi}(u, v)=u v+\theta(\max (u, v)) \phi(u) \phi(v),
$$


where $\phi$ and $\theta$ are two $I \rightarrow \mathbb{R}$ continuously differentiable functions. Remark that, if $\theta$ or $\phi$ is the null function on $I$ then $C_{\theta, \phi}=\Pi$, the independent copula. In the sequel, we thus assume that $\phi$ vanishes at most on isolated points of $I$, and that $\theta$ is not the zero function on $I$. The next theorem gives sufficient and necessary conditions on $\phi$ and $\theta$ to ensure that $C_{\theta, \phi}$ is a copula.

Theorem $1 C_{\theta, \phi}$ is a copula if and only if $\phi$ and $\theta$ satisfy the following conditions :

(a) $\phi(0)=0$,

(b) $\phi(1) \theta(1)=0$,

(c) $\phi^{\prime}(u)(\theta \phi)^{\prime}(v) \geq-1$ for all $0<u \leq v<1$,

(d) $\theta^{\prime}(u) \leq 0$ for all $u \in \stackrel{\circ}{I}=(0,1)$.

Proof: The proof involves four steps.

1. First, it is clear that $(\mathrm{P} 1) \Leftrightarrow(\mathrm{a})$ and $(\mathrm{P} 2) \Leftrightarrow(\mathrm{b})$.

2. Second, we show that $(\mathrm{P} 3) \Rightarrow(\mathrm{c})$. To this end, consider $0<u_{1}<u_{2} \leq v_{1}<v_{2}<1$. In this case, $\Delta\left(u_{1}, u_{2}, v_{1}, v_{2}\right)$ can be rewritten as

$$
\Delta\left(u_{1}, u_{2}, v_{1}, v_{2}\right)=\left(u_{2}-u_{1}\right)\left(v_{2}-v_{1}\right)+\left(\phi\left(u_{2}\right)-\phi\left(u_{1}\right)\right)\left(\theta\left(v_{2}\right) \phi\left(v_{2}\right)-\theta\left(v_{1}\right) \phi\left(v_{1}\right)\right),
$$

and thus $\Delta\left(u_{1}, u_{2}, v_{1}, v_{2}\right) \geq 0$ implies

$$
\frac{\phi\left(u_{2}\right)-\phi\left(u_{1}\right)}{u_{2}-u_{1}} \frac{\theta\left(v_{2}\right) \phi\left(v_{2}\right)-\theta\left(v_{1}\right) \phi\left(v_{1}\right)}{v_{2}-v_{1}} \geq-1 .
$$

Letting $u_{1} \rightarrow u_{2}^{-}$and $v_{1} \rightarrow v_{2}^{-}$in the previous inequality yields (c).

3. Similarly, we now show that (P3) $\Rightarrow(\mathrm{d})$. Taking $0<u<v<1$, we have

$$
\begin{aligned}
\Delta(u, v, u, v) & =(v-u)^{2}+\theta(v) \phi^{2}(v)+\theta(u) \phi^{2}(u)-2 \phi(u) \phi(v) \theta(v) \\
& =(v-u)\left[(v-u)+\theta(v) \phi(v) \frac{\phi(v)-\phi(u)}{v-u}-\phi(u) \frac{(\theta \phi)(v)-(\theta \phi)(u)}{v-u}\right] .
\end{aligned}
$$

Letting $u \rightarrow v^{-}$in the inequality $\Delta(u, v, u, v) \geq 0$ yields

$$
\theta(v) \phi(v) \phi^{\prime}(v)-\phi(v)(\theta \phi)^{\prime}(v) \geq 0,
$$

which is equivalent to $\phi^{2}(v) \theta^{\prime}(v) \leq 0$. This implies that $\theta^{\prime}(v) \leq 0$ for all $v \in I$ such that $\phi(v) \neq 0$. Since $\phi$ vanishes only on isolated points and $\theta^{\prime}$ is continuous, (d) is proved.

4. Finally, it remains to prove that (c, d) $\Rightarrow$ (P3). Let $\left(u_{1}, u_{2}, v_{1}, v_{2}\right) \in I^{4}$ such that $u_{1} \leq u_{2}$ and $v_{1} \leq v_{2}$. Let us denote by $R$ the rectangle $\left[u_{1}, u_{2}\right] \times\left[v_{1}, v_{2}\right]$, by $T$ the triangle with vertices $(0,0),(0,1)$ and $(1,1)$, and by $\bar{T}$ the triangle $\bar{T}=I^{2} \backslash T$. Suppose (c, d) hold and let us prove that $\Delta(R):=\Delta\left(u_{1}, u_{2}, v_{1}, v_{2}\right) \geq 0$. Three cases are considered.

(i) If $R \subset T$, i.e. $u_{2} \leq v_{1}$ then $\Delta\left(u_{1}, u_{2}, v_{1}, v_{2}\right)$ can be written as in (2.2) and the mean value theorem entails that there exist $u \in\left(u_{1}, u_{2}\right)$ and $v \in\left(v_{1}, v_{2}\right)$ such that

$$
\begin{aligned}
\Delta\left(u_{1}, u_{2}, v_{1}, v_{2}\right) & =\left(u_{2}-u_{1}\right)\left(v_{2}-v_{1}\right)\left[1+\frac{\phi\left(u_{2}\right)-\phi\left(u_{1}\right)}{u_{2}-u_{1}} \frac{\theta\left(v_{2}\right) \phi\left(v_{2}\right)-\theta\left(v_{1}\right) \phi\left(v_{1}\right)}{v_{2}-v_{1}}\right] \\
& =\left(u_{2}-u_{1}\right)\left(v_{2}-v_{1}\right)\left[1+\phi^{\prime}(u)(\theta \phi)^{\prime}(v)\right] \geq 0,
\end{aligned}
$$

as a consequence of (c).

(ii) If $R \subset \bar{T}$, then symmetry considerations show that $\Delta\left(u_{1}, u_{2}, v_{1}, v_{2}\right)=\Delta\left(v_{1}, v_{2}, u_{1}, u_{2}\right) \geq 0$ from the case (i). 
(iii) If $R \cap T \neq \emptyset$ and $R \cap \bar{T} \neq \emptyset$, then $R$ can be decomposed as non-overlapping rectangles $R=R_{1} \cup R_{2} \cup R_{3}$ such that $R_{1} \subset T$ or $R_{1} \subset \bar{T}, R_{2} \subset T$ or $R_{2} \subset \bar{T}$ and $R_{3}$ is a square of the form $[u, v] \times[u, v]$. Thus, $\Delta(R)=\Delta\left(R_{1}\right)+\Delta\left(R_{2}\right)+\Delta\left(R_{3}\right)$ and (i), (ii) entail that $\Delta\left(R_{1}\right) \geq 0$ and $\Delta\left(R_{2}\right) \geq 0$. Let us focus on $\Delta\left(R_{3}\right)$ :

$$
\begin{aligned}
\Delta(u, v, u, v) & =(v-u)^{2}+\theta(v) \phi(v)[\phi(v)-\phi(u)]-\phi(u)[\theta(v) \phi(v)-\theta(u) \phi(u)] \\
& =(v-u)^{2}+\int_{u}^{v}\left[\theta(v) \phi(v) \phi^{\prime}(t)-\phi(u)(\theta \phi)^{\prime}(t)\right] d t .
\end{aligned}
$$

Note that (c) implies that for all $0<t<v<1$,

$$
\int_{t}^{v} \phi^{\prime}(t)(\theta \phi)^{\prime}(y) d y \geq \int_{t}^{v}-1 d y=t-v,
$$

and thus $\phi^{\prime}(t)(\theta \phi)(v) \geq t-v+\theta(t) \phi(t) \phi^{\prime}(t)$. Similarly, (c) shows that for all $0<u<t<1$,

$$
(\theta \phi)^{\prime}(t) \int_{u}^{t} \phi^{\prime}(x) d x \geq u-t
$$

and thus $-(\theta \phi)^{\prime}(t) \phi(u) \geq u-t-(\theta \phi)^{\prime}(t) \phi(t)$. It follows that

$$
\Delta(u, v, u, v) \geq \int_{u}^{v}\left[(\theta \phi)(t) \phi^{\prime}(t)-\phi(t)(\theta \phi)^{\prime}(t)\right] d t=-\int_{u}^{v} \theta^{\prime}(t) \phi^{2}(t) d t \geq 0
$$

under condition (d). As a conclusion, $\Delta(R) \geq 0$ and (P3) is proved.

Note that (b) is true if $\phi(1)=0$ or $\theta(1)=0$. We refer to Section 5 for a detailed study of the corresponding sub-families. Although the copula $C_{\theta, \phi}$ has full support $I^{2}$, the following proposition shows that, in general, it is neither absolutely continuous, nor singular.

Proposition 1 The copula $C_{\theta, \phi}$ has both absolutely continuous and singular components $A_{\theta, \phi}$ and $S_{\theta, \phi}$, respectively, given by

$$
A_{\theta, \phi}(u, v)=u v+\theta(\max (u, v)) \phi(u) \phi(v)+\int_{0}^{\min (u, v)}\left(\theta^{\prime} \phi^{2}\right)(t) d t
$$

and

$$
S_{\theta, \phi}(u, v)=-\int_{0}^{\min (u, v)}\left(\theta^{\prime} \phi^{2}\right)(t) d t .
$$

Proof: The absolutely continuous component of $C_{\theta, \phi}$ is given by

$$
A_{\theta, \phi}(u, v)=\int_{0}^{u} \int_{0}^{v} \frac{\partial^{2}}{\partial s \partial t} C_{\theta, \phi}(s, t) d t d s
$$

with

$$
\frac{\partial^{2}}{\partial s \partial t} C_{\theta, \phi}(s, t)=1+(\theta \phi)^{\prime}(\max (s, t)) \phi^{\prime}(\min (s, t)) .
$$

Assume for instance $v \geq u$. Then, $A_{\theta, \phi}$ can be written as

$$
\begin{aligned}
A_{\theta, \phi}(u, v) & =u v+\int_{0}^{u} \int_{0}^{s}(\theta \phi)^{\prime}(s) \phi^{\prime}(t) d t d s+\int_{0}^{u} \int_{s}^{v}(\theta \phi)^{\prime}(t) \phi^{\prime}(s) d t d s \\
& =u v+(\theta \phi)(v) \phi(u)+\int_{0}^{u}\left(\theta^{\prime} \phi^{2}\right)(s) d s \\
& =C_{\theta, \phi}(u, v)+\int_{0}^{u}\left(\theta^{\prime} \phi^{2}\right)(s) d s,
\end{aligned}
$$

and the conclusion follows. The case $v<u$ is similar. 
Thus, the mass of the singular component is concentrated on the first diagonal of the square $I^{2}$. Denoting by $(U, V)$ a uniform random pair on $I^{2}$ with copula $C_{\theta, \phi}$, we have

$$
\mathbb{P}(U=V)=-\int_{0}^{1}\left(\theta^{\prime} \phi^{2}\right)(t) d t .
$$

Besides, the copula $C_{\theta, \phi}$ has no singular component if and only if $\theta$ is a constant function. This case is described more precisely in Section 5 .

\section{Measures of association}

In the next two sections, we note $(X, Y)$ a random pair with joint cdf $H$, copula $C$ and margins $F$ and $G$. The case $C=C_{\theta, \phi}$ is explicitly precised.

\subsection{Spearman's Rho}

Several invariant to strictly increasing function measures of association between the components of the random pair $(X, Y)$ can be considered: the normalized volume between graphs of $H$ and $F G$ [28], Kendall's Tau [22], paragraph 5.1.1, Gini's coefficient [22], Blomqwist's medial correlation coefficient [22], paragraph 5.1.4, and Spearman's Rho [22], paragraph 5.1.2, which is the probability of concordance minus the probability of discordance of two random pairs with joint cdf $H$ and $F G$. Here, we focus on this latter measure, showing in Subsection 5.3 that this measure can achieve any value in $[-3 / 4,1]$. A first step towards this result consists in remarking that Spearman's Rho can be written only in terms of the copula $C$ :

$$
\rho=12 \int_{0}^{1} \int_{0}^{1} C(u, v) d u d v-3
$$

Note that $\rho$ coincides with the correlation coefficient between the uniform marginal distributions. In the case of a copula generated by (2.1), it can be expressed thanks to the functions $\phi$ and $\theta$.

Proposition 2 Let $(X, Y)$ be a random pair with copula $C_{\theta, \phi}$. The Spearman's Rho is given by

$$
\rho_{\theta, \phi}=12\left[\Phi^{2}(1) \theta(1)-\int_{0}^{1} \Phi^{2}(t) \theta^{\prime}(t) d t\right],
$$

where $\Phi(t)=\int_{0}^{t} \phi(x) d x$.

Proof: Clearly, Spearman's Rho can be expanded as

$$
\rho_{\theta, \phi}=12\left[\int_{0}^{1} \int_{v}^{1} \phi(u) \phi(v) \theta(u) d u d v+\int_{0}^{1} \int_{0}^{v} \phi(u) \phi(v) \theta(v) d u d v\right] .
$$

An integration by parts shows that both terms are equal and thus,

$$
\begin{aligned}
\rho_{\theta, \phi} & =24 \int_{0}^{1}\left(\phi(v) \theta(v) \int_{0}^{v} \phi(u) d u\right) d v \\
& =24 \int_{0}^{1} \theta(v) \phi(v) \Phi(v) d v \\
& =12\left[\Phi^{2}(1) \theta(1)-\int_{0}^{1} \Phi^{2}(t) \theta^{\prime}(t) d t\right],
\end{aligned}
$$

by a new integration by parts. 


\subsection{Tail dependence}

The upper tail dependence can be quantified by the upper tail dependence coefficient [13], paragraph 2.1.10, defined as:

$$
\lambda=\lim _{t \rightarrow 1^{-}} \mathbb{P}(F(X)>t \mid G(Y)>t) .
$$

Again, this coefficient can be written only in terms of the copula $C$ (see [22, Theorem 5.4.2):

$$
\lambda=\lim _{u \rightarrow 1^{-}} \frac{\bar{C}(u, u)}{1-u},
$$

where $\bar{C}$ is the survival copula, i.e. $\bar{C}(u, v)=1-u-v+C(u, v)$. In our family, the following simplified expression can be obtained:

Proposition 3 Let $(X, Y)$ be a random pair with copula $C_{\theta, \phi}$. The upper tail dependence coefficient is: $\lambda_{\theta, \phi}=-\phi^{2}(1) \theta^{\prime}(1)$.

Proof: Clearly, the upper tail dependence coefficient can be simplified as

$$
\lambda_{\theta, \phi}=\lim _{u \rightarrow 1^{-}} \frac{\phi^{2}(u) \theta(u)}{1-u} .
$$

Taking into account of (b) yields

$$
\lambda_{\theta, \phi}=-\lim _{u \rightarrow 1^{-}} \frac{\phi^{2}(1) \theta(1)-\phi^{2}(u) \theta(u)}{1-u}=-\left(\phi^{2} \theta\right)^{\prime}(1)=-\phi^{2}(1) \theta^{\prime}(1),
$$

and the result is proved.

Note that a coefficient measuring the lower tail dependence can also be defined as,

$$
\lim _{t \rightarrow 0^{+}} \mathbb{P}(F(X) \leq t \mid G(Y) \leq t) .
$$

but it is always zero in the considered family.

\section{Concepts of dependence}

In this section, for the sake of simplicity, we assume that $X$ any $Y$ are exchangeable. Several concepts of positive dependence have been introduced and characterized in terms of copulas. $X$ and $Y$ are

- Positively Quadrant Dependent (PQD) if $\mathbb{P}(X \leq x, Y \leq y) \geq \mathbb{P}(X \leq x) \mathbb{P}(Y \leq y)$, for all $(x, y) \in \mathbb{R}^{2}$ or equivalently

$$
\forall(u, v) \in I^{2}, \quad C(u, v) \geq u v .
$$

- Left Tail Decreasing (LTD) if $\mathbb{P}(Y \leq y \mid X \leq x)$ is non-increasing in $x$ for all $y$, or equivalently, see Theorem 5.2.5 in [22], $u \rightarrow C_{\theta, \phi}(u, v) / u$ is non-increasing for all $v \in I$.

- Right Tail Increasing (RTI) if $\mathbb{P}(Y>y \mid X>x)$ is non-decreasing in $x$ for all $y$ or, equivalently, $u \rightarrow\left(v-C_{\theta, \phi}(u, v)\right) /(1-u)$ is non-increasing for all $v \in I$.

- Left Corner Set Decreasing (LCSD) if $\mathbb{P}\left(X \leq x, Y \leq y \mid X \leq x^{\prime}, Y \leq y^{\prime}\right)$ is non-increasing in $x^{\prime}$ and $y^{\prime}$ for all $x$ and $y$, or equivalently, see Corollary 5.2.17 in [22, $C$ is a totally positive function of order 2 , i.e. for all $\left(u_{1}, u_{2}, v_{1}, v_{2}\right) \in I^{4}$ such that $u_{1} \leq u_{2}$ and $v_{1} \leq v_{2}$, one has

$$
C\left(u_{1}, v_{1}\right) C\left(u_{2}, v_{2}\right)-C\left(u_{1}, v_{2}\right) C\left(u_{2}, v_{1}\right) \geq 0
$$


- Right Corner Set Increasing (RCSI) if $\mathbb{P}\left(X>x, Y>y \mid X>x^{\prime}, Y>y^{\prime}\right)$ is non-decreasing in $x^{\prime}$ and $y^{\prime}$ for all $x$ and $y$, or equivalently, the survival copula $\hat{C}$ associated to $\mathrm{C}$ is a totally positive function of order 2 .

Concepts of negative dependence can be similarly defined. Recall that $\theta$ is supposed not to be the null function on $I$ and introduce

$$
v^{*}=\sup \{v \in I ; \theta(v) \neq 0\} .
$$

The point $v^{*}$, which can be seen as the endpoint of $\theta$, plays a central role in the dependence properties of the copula $C_{\theta, \phi}$, see Theorem 2 below.

Theorem 2 Let $(X, Y)$ a random pair with copula $C_{\theta, \phi} . X$ and $Y$ are

(i) $P Q D$ if and only if $\theta(u) \geq 0$ for all $u \in I$ and $\phi$ has a constant sign on $\left[0, v^{*}\right]$.

(ii) LTD if and only if $\theta(u) \geq 0$ for all $u \in I$ and $u \rightarrow \phi(u) / u$ and $u \rightarrow \theta(u) \phi(u) / u$ are either both non-increasing or both non-decreasing on $\left[0, v^{*}\right]$.

(iii) RTI if and only if $\theta(u) \geq 0$ for all $u \in I$ and $u \rightarrow \phi(u) /(1-u)$ and $u \rightarrow \theta(u) \phi(u) /(1-u)$ are either both non-increasing or both non-decreasing on $\left[0, v^{*}\right]$.

(iv) LCSD if and only if they are LTD.

(v) RCSI if and only if they are RTI.

Proof: (i): Condition (4.1) can be rewritten as

$$
\theta(\max (u, v)) \phi(u) \phi(v) \geq 0, \quad \forall(u, v) \in I^{2} .
$$

Suppose first that $(X, Y)$ is PQD. Considering $u=v$ in (4.3) shows that $\theta(u) \phi^{2}(u) \geq 0$ for all $u \in I$. Since $\phi$ vanishes at most on isolated points, $\theta(u) \geq 0$ almost everywhere on $I$. Recalling that $\theta$ is continuous on $I$, we have $\theta(u) \geq 0$ for all $u \in I$. Moreover, from $(\mathrm{d}), \theta$ in non-increasing on $I$, and consequently $\theta(t)>0$ for all $t \in\left[0, v^{*}\right)$. Thus, for all $(u, v) \in\left[0, v^{*}\right)^{2}, \theta(\max (u, v))>0$ and condition (4.3) yields $\phi(u) \phi(v) \geq 0$ which implies that $\phi$ has a constant sign on $\left[0, v^{*}\right]$.

Conversely, suppose $\theta(u) \geq 0$ for all $u \in I$ and $\phi$ has a constant sign on $\left[0, v^{*}\right]$. For symmetry reasons, it suffices to verify condition (4.3) for $0 \leq u \leq v \leq 1$. In this case $\theta(v) \phi(u) \phi(v)=0$ if $v \geq v^{*}$ and $\theta(v) \phi(u) \phi(v) \geq 0$ otherwise.

(ii) and (iii): Proofs are similar. Focusing on (iii), the necessary and sufficient condition can be rewritten as

$$
u \rightarrow \theta(\max (u, v)) \phi(v) \phi(u) /(1-u) \text { is non-decreasing for all } v \in\left[0, v^{*}\right] .
$$

Supposing that $(X, Y)$ is RTI also implies that $(X, Y)$ is PQD and consequently $\theta(u) \geq 0$ for all $u \in I$ and $\phi$ has a constant sign on $\left[0, v^{*}\right]$. Assuming for example that $\phi(u) \geq 0$ for all $u \in\left[0, v^{*}\right]$, condition (4.4) implies that $u \rightarrow \phi(u) /(1-u)$ is non-decreasing on $[0, v]$ and that $u \rightarrow \theta(u) \phi(u) /(1-u)$ is non-decreasing on $\left[v, v^{*}\right]$, for all $v \in\left[0, v^{*}\right]$. Thus, $u \rightarrow \phi(u) /(1-u)$ and $u \rightarrow \theta(u) \phi(u) /(1-u)$ are both non-decreasing on $\left[0, v^{*}\right]$. Conversely, assume $\theta(u) \geq 0$ for all $u \in I$, and that $u \rightarrow \phi(u) /(1-u)$ and $u \rightarrow \theta(u) \phi(u) /(1-u)$ are non-decreasing on $\left[0, v^{*}\right]$. From Lemma 1(i) in the appendix, $\phi$ is non-negative on $\left[0, v^{*}\right]$, and (4.4) is clearly true.

(iv) and (v): Proofs are similar. Let us focus on (iv). It is well-known that $(X, Y)$ LCSD implies $(X, Y)$ LTD. Let us prove that the converse result is also true in the $C_{\theta, \phi}$ family. Suppose $(X, Y)$ is LTD. Following (ii), one can assume that $u \rightarrow \phi(u) / u$ is non-increasing and $\phi(u) \geq 0$ for all $u \in\left[0, v^{*}\right]$, together with $\theta(u) \geq 0$ for all $u \in I$. Lemma 1(ii) entails that $u \rightarrow \theta(u) \phi(u) / u$ 
is non-increasing on $\left[0, v^{*}\right]$. Four cases have to be considered to prove (4.2):

- If $0 \leq u_{1} \leq u_{2} \leq v_{1} \leq v_{2} \leq 1$, condition (4.2) reduces to $A_{1} \geq 0$, where we have defined

$$
A_{1}:=\left[\frac{\theta\left(v_{1}\right) \phi\left(v_{1}\right)}{v_{1}}-\frac{\theta\left(v_{2}\right) \phi\left(v_{2}\right)}{v_{2}}\right]\left[\frac{\phi\left(u_{1}\right)}{u_{1}}-\frac{\phi\left(u_{2}\right)}{u_{2}}\right] .
$$

If $u_{2} \leq v^{*}$, Lemma 2 in the appendix yields $A_{1} \geq 0$. Otherwise, $u_{2}>v^{*}$ implies $v_{1} \geq v^{*}$ and Lemma 2 yields $A_{1}=0$.

- If $0 \leq u_{1} \leq v_{1} \leq u_{2} \leq v_{2} \leq 1$, condition (4.2) can be rewritten $A_{2} \geq 0$, with

$$
A_{2}:=A_{1}+C_{\theta, \phi}\left(u_{1}, v_{2}\right)\left[\theta\left(v_{1}\right)-\theta\left(u_{2}\right)\right] \frac{\phi\left(u_{2}\right) \phi\left(v_{1}\right)}{u_{1} u_{2} v_{1} v_{2}} .
$$

If $u_{2} \leq v^{*}$, then $\phi\left(u_{2}\right) \phi\left(v_{1}\right) \geq 0$ and Lemma 2 yields $A_{1} \geq 0$. Consequently, $A_{2} \geq 0$.

If $v_{1} \leq v^{*} \leq u_{2}$, then $\theta\left(u_{2}\right)=\theta\left(v_{2}\right)=0$ and $A_{2}$ reduces to

$$
A_{2}=\frac{\theta\left(v_{1}\right) \phi\left(v_{1}\right) \phi\left(u_{1}\right)}{u_{1} v_{1}} \geq 0 .
$$

Finally, if $v^{*} \leq v_{1}$, then $A_{2}=0$.

- If $0 \leq u_{1} \leq v_{1} \leq v_{2} \leq u_{2} \leq 1$, condition (4.2) can be rewritten $A_{3} \geq 0$, with

$$
\begin{aligned}
A_{3}:=A_{1}+\frac{\phi\left(u_{2}\right)}{u_{2}} \quad & {\left[\frac{\phi\left(v_{1}\right)}{v_{1}} \frac{C_{\theta, \phi}\left(u_{1}, v_{2}\right)}{u_{1} v_{2}}\left(\theta\left(v_{1}\right)-\theta\left(u_{2}\right)\right)+\frac{\phi\left(v_{2}\right)}{v_{2}} \frac{C_{\theta, \phi}\left(u_{1}, v_{1}\right)}{u_{1} v_{1}}\left(\theta\left(u_{2}\right)-\theta\left(v_{2}\right)\right)\right] } \\
=A_{1}+\frac{\phi\left(u_{2}\right)}{u_{2}} \quad & {\left[\left(\theta\left(v_{1}\right)-\theta\left(u_{2}\right)\right)\left(\frac{\phi\left(v_{1}\right)}{v_{1}}-\frac{\phi\left(v_{2}\right)}{v_{2}}\right)\right.} \\
& \left.+\left(\theta\left(v_{1}\right)-\theta\left(v_{2}\right)\right) \frac{\phi\left(v_{2}\right)}{v_{2}}\left(1+\theta\left(u_{2}\right) \frac{\phi\left(u_{1}\right)}{u_{1}} \frac{\phi\left(v_{1}\right)}{v_{1}}\right)\right] .
\end{aligned}
$$

If $u_{2} \leq v^{*}$, then Lemma 2 yields $A_{1} \geq 0$ and all the above differences are non-negative. Consequently, $A_{3} \geq 0$.

If $v_{2} \leq v^{*} \leq u_{2}$, then $\theta\left(u_{2}\right)=0$ and $A_{3}$ reduces to

$$
A_{3}=\left(\frac{\theta\left(v_{1}\right) \phi\left(v_{1}\right)}{v_{1}}-\frac{\theta\left(v_{2}\right) \phi\left(v_{2}\right)}{v_{2}}\right) \frac{\phi\left(u_{1}\right)}{u_{1}} \geq 0 .
$$

If $v_{1} \leq v^{*} \leq v_{2}$, then $\theta\left(u_{2}\right)=\theta\left(v_{2}\right)=0$ and $A_{3}$ reduces to

$$
A_{3}=\frac{\theta\left(v_{1}\right) \phi\left(v_{1}\right)}{v_{1}} \frac{\phi\left(u_{1}\right)}{u_{1}} \geq 0 .
$$

Finally, if $v^{*} \leq v_{1}$, then $A_{3}=0$.

- The three remaining situations are equivalent to the three previous ones since the considered copulas are symmetric in the arguments.

\section{$5 \quad$ Sub-families and examples}

Recall that (b) is true if $\phi(1)=0$ or $\theta(1)=0$. The corresponding sub-families are now studied in details and examples of copulas in each sub-family are given. 


\subsection{The case $\theta(1)=0$}

Let us focus on the sub-family of $C_{\theta, \phi}$ defined by conditions (a), (b1), (c) and (d), where (b1) $\theta(1)=0$

First, note that (b1, d) implies that $\theta$ is non negative on $I$. From Proposition 2 and (b1), Spearman's Rho is given by

$$
\rho_{\theta, \phi}=-12 \int_{0}^{1} \Phi^{2}(t) \theta^{\prime}(t) d t
$$

and (d) entails that, in this sub-family, $\rho_{\theta, \phi} \geq 0$. Second, we focus on copulas generated by univariate cdf and defined by

$$
C_{\bar{K}^{-1}, I d}(u, v)=u v\left[1+\bar{K}^{-1}(\max (u, v))\right],
$$

where $K$ is a cdf on $\mathbb{R}^{+}, \bar{K}$ is the associated survival function, $\bar{K}^{-1}$ is its generalized inverse defined as $\bar{K}^{-1}(x)=K^{-1}(1-x)=\inf \{t \geq 0, K(t) \geq 1-x\}$ and $\phi=I d$ is the identity function. We assume that $K$ is strictly increasing and differentiable on $\left(K^{-1}(0), K^{-1}(1)\right)$, the associated point distribution function is denoted by $k$. The following corollary provides sufficient and necessary conditions to ensure that $C_{\bar{K}^{-1}, I d}$ is a copula. It shows that the hazard function $k / \bar{K}$ is the key quantity in this context.

Corollary $1 C_{\bar{K}^{-1}, I d}$ is a copula if and only if, for all $t \geq 0$ such that $0<K(t)<1$,

$$
\frac{k(t)}{\bar{K}(t)} \geq \frac{1}{1+t}
$$

Proof: Condition (a) is verified since $\phi(x)=x$. Besides, $K(0)=0$ is equivalent to condition (b). Condition (c) is equivalent to

$$
\frac{x}{k\left(\bar{K}^{-1}(x)\right)}-\bar{K}^{-1}(x) \leq 1
$$

for all $x \in I$, which can be rewritten as

$$
\frac{\bar{K}(t)}{k(t)}-t \leq 1,
$$

for all $t \geq 0$ by introducing $t=\bar{K}^{-1}(x)$. The conclusion follows from Theorem 1 ,

As a consequence of condition (5.2), one can easily show that necessarily, $\bar{K}(x) \leq 1 /(1+x)$. Let us also note that, from (5.1) and Proposition 3, Spearman's Rho as well as the upper tail dependence coefficient can be rewritten in terms of $K$ as

$$
\rho_{\bar{K}^{-1}, I d}=3 \int_{0}^{+\infty} \bar{K}^{4}(t) d t \text { and } \lambda_{\bar{K}^{-1}, I d}=1 / k(0) .
$$

In this sub-family, Blomqwvist's medial correlation coefficient $\beta$ benefits of a nice interpretation

$$
\beta_{\bar{K}^{-1}, I d}=4 C_{\bar{K}^{-1}, I d}(1 / 2,1 / 2)-1=\bar{K}^{-1}(1 / 2)=K^{-1}(1 / 2),
$$

as the median of the cdf $K$. Besides, characterizations of dependence properties in Proposition 2 can be simplified as 
Corollary 2 Let $(X, Y)$ a random pair with copula $C_{\bar{K}^{-1}, I d} . X$ and $Y$ are always $P Q D, L T D$ and LCSD. Moreover, $X$ and $Y$ are RTI and RCSI if and only if for all $t>0$ such that $0<K(t)<1$,

$$
\frac{k(t)}{K(t) \bar{K}(t)} \geq \frac{1}{t}
$$

The proof is similar to the one of Corollary 1, In examples $1, \ldots 4$, all the PQD, LTD, LCSD, RTI and RCSI properties hold.

\section{Examples}

1. A first example of copula belonging to this sub-family is the Cuadras-Augé copula [4]:

$$
C_{\alpha}^{\mathrm{CA}}(u, v)=\min (u, v)^{\alpha}(u v)^{1-\alpha}=M^{\alpha}(u, v) \Pi^{1-\alpha}(u, v)
$$

where $\alpha \in[0,1], M$ is the Fréchet upper bound defined by $M(u, v)=\min (u, v)$ and $\Pi$ is the product copula $\Pi(u, v)=u v$. The copula $C_{\alpha}^{\mathrm{CA}}$ can be interpreted as the weighted geometric mean of $M$ and $\Pi$. It is generated by the $C_{\bar{K}^{-1}, I d}$ family with $\bar{K}(x)=(1+x)^{-1 / \alpha}$, which is the survival function of a Generalized Pareto Distribution (GPD) with positive shape parameter $1 / \alpha$ (see for instance Table 1.2.6 in [5]). The associated Spearman's Rho given by (5.3) is $\rho_{\alpha}^{\mathrm{CA}}=3 \alpha /(4-\alpha)$ and the upper tail dependence coefficient is $\lambda_{\alpha}^{\mathrm{CA}}=\alpha$.

2. Another similar example is the family (B11), introduced in [13], page 148:

$$
C_{\sigma}^{\mathrm{B} 11}(u, v)=\sigma \min (u, v)+(1-\sigma) u v=\sigma M(u, v)+(1-\sigma) \Pi(u, v),
$$

where $\sigma \in(0,1]$. The copula $C_{\sigma}^{\text {B11 }}$ can be interpreted as the weighted arithmetic mean of $M$ and $\Pi$. It is generated by the $C_{\bar{K}^{-1}, I d}$ family with $\bar{K}(x)=(1+x / \sigma)^{-1}$, which is the survival function of a GPD with scale parameter $\sigma$ (see for instance Table 1.2.6 in [5]). The associated Spearman's Rho and upper tail dependence coefficient are $\rho_{\sigma}^{\mathrm{B} 11}=\lambda_{\sigma}^{\mathrm{B} 11}=\sigma$. Note that, for all $\alpha \in(0,1]$, one always has $\rho_{\alpha}^{\mathrm{B} 11} \geq \rho_{\alpha}^{\mathrm{CA}}$. Since both Cuadras-Augé and (B11) copulas are indexed by a single parameter they do not allow the pair $(\rho, \lambda)$ to reach arbitrary values in $[0,1]^{2}$.

3. To partially overcome this limitation, it is natural to consider $\bar{K}(x)=(1+x / \sigma)^{-1 / \alpha}$, which is the survival function of a GPD with positive shape parameter $1 / \alpha$ and scale parameter $\sigma, \alpha \in(0,1], \alpha \sigma \in(0,1]$. The associated Spearman's Rho given by (5.3) is $\rho_{\alpha, \sigma}^{\mathrm{GPD}}=3 \alpha \sigma /(4-\alpha)$ and the upper tail dependence coefficient $\lambda_{\alpha, \sigma}^{\mathrm{GPD}}=\alpha \sigma$. Thus, the $C_{\alpha, \beta}^{\mathrm{GPD}}$ copula allows the pair $(\rho, \lambda)$ to reach any value in the triangle $\left\{(\rho, \lambda) \in(0,1)^{2}: \rho \leq \lambda<4 \rho / 3\right\}$ with the following choice of parameters: $\alpha(\lambda, \rho)=4-3 \lambda / \rho$ and $\sigma(\lambda, \rho)=(\rho \lambda) /(4 \rho-3 \lambda)$.

4. Choosing $K$ as the cdf of the uniform distribution on $[0, \alpha], \alpha \leq 1$ gives rise to the family of copulas

$$
C_{\alpha}^{\text {Uniform }}(u, v)=u v(1+\alpha \min (1-u, 1-v)),
$$

introduced in [11], Section 1, and with associated Spearman's Rho $\rho_{\alpha}^{\text {Uniform }}=3 \alpha / 5$ and upper tail dependence coefficient $\lambda_{\alpha}^{\text {Uniform }}=\alpha$.

5. Finally, note that the family

$$
C_{f}(u, v)=\min (u, v) f(\max (u, v))
$$

proposed in [6] can also enter our sub-family with an appropriate choice of $K$. Basing on Example 1, we can state the following result:

Proposition 4 Suppose $C_{\theta, \phi}$ is a copula and $\theta(1)=0$. Thus, $0 \leq \rho_{\theta, \phi} \leq 1$ and $0 \leq \lambda_{\theta, \phi} \leq 1$, and these bounds are reached within the sub-family. 


\subsection{The case $\phi(1)=0$}

Here, we focus on the sub-family of $C_{\theta, \phi}$ defined by conditions (a), (b2), (c) and (d), where (b2) $\phi(1)=0$,

Note that (b2) implies that the upper tail dependence coefficient is always null in this sub-family. This sub-family encompasses the semiparametric family of copulas with constant function $\theta$ defined in (1.7). Consequently, this sub-family also includes the FGM family (1.1), the parametric family of symmetric copulas with cubic sections proposed in [21, equation (4.4), both kernel families (1.3) and (1.4) introduced in [11], and the PQD copulas (1.5) introduced in [15].

From Proposition 2, in the subfamily of $C_{\theta, \phi}$ constrained by (b2), the following lower bound for Spearman's Rho holds:

$$
\rho_{\theta, \phi} \geq 12 \Phi^{2}(1) \theta(1)
$$

where the right-hand term can be interpreted as Spearman's Rho associated to the copula (1.7) with constant function $\theta()=.\theta(1)$. Since, in this particular case, Spearman's Rho is lower bounded by $-3 / 4$ (see [1], Proposition 2), we have:

Proposition 5 Suppose $C_{\theta, \phi}$ is a copula and $\phi(1)=0$. Thus, $\lambda_{\theta, \phi}=0$ and $\rho_{\theta, \phi} \geq-3 / 4$, and this bound is reached within the subfamily.

Remark. It is of course possible to build copulas such that $\phi(1)=0$ and $\theta$ is a non constant function. As an example, consider the function $\phi(x)=x(1-x)$ which generates the FGM family of copulas. Taking $u=0$ in condition (c) and integrating with respect to $v \in[x, 1]$ imply that $\theta(x) \leq 1 / x$ for all $0<x \leq 1$. Let us consider the extreme case $\theta(x)=1 / x$. The copula writes

$$
C(u, v)=\Pi(u, v)+(1-u)(1-v) M(u, v),
$$

and the associated Spearman's Rho is $\rho=3 / 5$ which is much larger than the maximum value $(\rho=1 / 3)$ in the FGM family.

\subsection{General case}

Collecting Proposition 4 and Proposition 5, we are now in position to provide the bounds for the general family (2.1).

Proposition 6 Suppose $C_{\theta, \phi}$ is a copula. Thus, $0 \leq \lambda_{\theta, \phi} \leq 1$ and $-3 / 4 \leq \rho_{\theta, \phi} \leq 1$, and these bounds are reached within the family.

Besides, Proposition 1 entails that the copulas (1.7) are the only ones which are absolutely continuous. Thus, we can conclude that, in the general $C_{\theta, \phi}$ family, the absolute continuity is incompatible with the upper tail dependence.

\section{References}

[1] Amblard, C. and Girard, S., 2002. Symmetry and dependence properties within a semiparametric family of bivariate copulas. Nonparametric Statistics, 14(6), 715-727.

[2] Amblard, C. and Girard, S., 2005. Estimation procedures for a semiparametric family of bivariate copulas. Journal of Computational and Graphical Statistics, 14(2), 1-15.

[3] Bairamov, I. and Kotz, S., 2002. Dependence structure and symmetry of Huang-Kotz FGM distributions and their extensions. Metrika, 56, 55-72. 
[4] Cuadras, C. M., Augé, J., 1981. A continuous general multivariate distribution and its properties, Communication in Statistics - Theory and Methods, 10, 339-353.

[5] Embrechts, P., Klüppelberg, C., Mikosch, T., 1997. Modelling extremal events, Springer.

[6] Durante, F., 2006. A new class of symmetric bivariate copulas. Nonparametric Statistics, 18, 499-510.

[7] Farlie, D. G. J., 1960. The performance of some correlation coefficients for a general bivariate distribution. Biometrika, 47, 307-323.

[8] Fischer, M. and Klein, I., 2007. Constructing Symmetric Generalized FGM Copulas by means of certain Univariate Distributions, Metrika, 65, 243-260.

[9] Genest, C. and MacKay, J., 1986. Copules archimédiennes et familles de lois bidimensionnelles dont les marges sont données. Canad. J. Statist., 14, 145-159.

[10] Gumbel, E. J., 1960. Bivariate Exponential distributions. Journal of the American Statistical Association, 55, 698-707.

[11] Huang, J. S. and Kotz, S., 1999. Modifications of the Farlie-Gumbel-Morgenstern distribution. A tough hill to climb. Metrika, 49, 135-145.

[12] Huang, J. S. and Kotz, S., 1984. Correlation structure in iterated Farlie-GumbelMorgenstern distributions. Biometrika, 71, 633-636.

[13] Joe, H., 1997. Multivariate models and dependence concepts. Monographs on statistics and applied probability, 73, Chapman \& Hall.

[14] Kim, J.-M. and Sungur, E. A., 2004. New class of bivariate copulas. Proceedings for the Spring Conference 2004, Korean Statistical Society, 207-212.

[15] Lai, C. D. and Xie, M., 2000. A new family of positive quadrant dependence bivariate distributions. Statistics and Probability Letters, 46, 359-364.

[16] Lee, M. T., 1996. Properties and applications of the Sarmanov family of bivariate distributions. Communication in Statistics - Theory and Methods, 25(6), 1207-1222.

[17] Lehmann, E. L., 1966. Some concepts of dependence. Ann. Math. Statist., 37, 1137-1153.

[18] Morgenstern, D., 1956. Einfache Beispiele zweidimensionaler Verteilungen. Mitteilungsblatt für Mathematische Statistik, 8, 234-235.

[19] Nelsen, R. B., 1991. Copulas and association. Advances and probability distribution with given marginals. Dall'Aglio, G., Kotz, S. and Salineti, G. eds Kluwer academic Publishers, Dordrecht.

[20] Nelsen, R. B., 1993. Some concepts of bivariate symmetry. Nonparametric Statistics, 3, 95-101.

[21] Nelsen, R. B., Quesada-Molina, J. J. and Rodríguez-Lallena, J. A., 1997. Bivariate copulas with cubic sections. Nonparametric Statistics, 7, 205-220.

[22] Nelsen, R. B., 2006. An introduction to copulas, Second Edition. Springer Series in Statistics, Springer. 
[23] Quesada-Molina, J. J. and Rodríguez-Lallena, J. A., 1995. Bivariate copulas with quadratic sections. Nonparametric Statistics, 5, 323-337.

[24] Rodríguez-Lallena, J. A., 1992. Estudio de la compabilidad y diseño de nuevas familias en la teoria de cópulas. Aplicaciones. Tesis doctoral, Universidad de Granada.

[25] Rodríguez-Lallena, J. A. and Úbeda-Flores, M., 2004. A new class of bivariate copulas. Statistics and Probability Letters, 9(5), 315-325.

[26] Sarmanov, O. V., 1966. Generalized normal correlation and two-dimensional Fréchet classes. Doklady Akademii Nauk SSSR, 168(1), 596-599.

[27] Shubina, M. and Lee, M. T., 2004. On maximum attainable correlation and other measures of dependence for the Sarmanov family of bivariate distributions. Communication in Statistics - Theory and Methods, 33(5), 1031-1052.

[28] Schweizer, B. and Wolff, E. F, 1981. On nonparametric measures of dependence for random variables. The Annals of Statistics, 9, 879-885.

[29] Sklar, A., 1959. Fonctions de répartition à $n$ dimensions et leurs marges. Publ. Inst. Statist. Univ. Paris, 8, 229-231.

\section{Appendix: Auxiliary lemmas}

Lemma 1 Assume $C_{\theta, \phi}$ is a copula.

(i) If $u \rightarrow \phi(u) /(1-u)$ is non-decreasing (resp. non-increasing) on $\left[0, v^{*}\right]$ then $\phi(u) \geq 0$ $($ resp. $\leq 0)$ for all $u \in\left[0, v^{*}\right]$.

If, moreover, $\theta(u) \geq 0$ for all $u \in I$, then

(ii) If $\phi(u) \leq 0$ (resp. $\geq 0$ ) for all $u \in J \subset I$ and $u \rightarrow \phi(u) / u$ is non-decreasing (resp. non-increasing) on $J$ then $u \rightarrow \theta(u) \phi(u) / u$ is non-decreasing (resp. non-increasing) on $J$.

\section{Proof:}

(i) Assume $u \rightarrow \phi(u) /(1-u)$ is non-decreasing on $\left[0, v^{*}\right]$. Then, from (a), $\forall u \in\left[0, v^{*}\right]$, $\phi(u) /(1-u) \geq \phi(0)=0$. Therefore, $\phi(u) /(1-u)$ is non-negative on $\left[0, v^{*}\right]$ and the conclusion follows.

(ii) Remark that $[\theta(u) \phi(u) / u]^{\prime}=\theta^{\prime}(u) \phi(u) / u+\theta(u)[\phi(u) / u]^{\prime}$. Thus, if $\phi(u) \leq 0, \theta(u) \geq 0$ and $u \rightarrow \phi(u) / u$ is non-decreasing, (d) implies that $[\theta(u) \phi(u) / u]^{\prime} \geq 0$ for all $u \in J$.

Lemma 2 Assume $C_{\theta, \phi}$ is a copula, $\theta(u) \geq 0$ for all $u \in I$ and

(i) either $\{u \rightarrow \phi(u) / u$ is non-increasing and $\phi(u) \geq 0\}$ for all $u \in\left[0, v^{*}\right]$,

(ii) or $\{u \rightarrow \phi(u) / u$ is non-decreasing and $\phi(u) \leq 0\}$ for all $u \in\left[0, v^{*}\right]$.

Let $\left(u_{1}, u_{2}, v_{1}, v_{2}\right) \in I^{4}$ such that $u_{1} \leq u_{2}, v_{1} \leq v_{2}$ and introduce

$$
A_{1}:=\left[\frac{\theta\left(v_{1}\right) \phi\left(v_{1}\right)}{v_{1}}-\frac{\theta\left(v_{2}\right) \phi\left(v_{2}\right)}{v_{2}}\right]\left[\frac{\phi\left(u_{1}\right)}{u_{1}}-\frac{\phi\left(u_{2}\right)}{u_{2}}\right] .
$$

Then, $u_{2} \leq v^{*}$ entails $A_{1} \geq 0$ and $v^{*} \leq v_{1}$ entails $A_{1}=0$. 
Proof: Assume (i) holds, situation (ii) is similar. First, remark that $u \rightarrow \theta(u) \phi(u) / u$ is nonincreasing on the whole $I$ interval, since this function is non-negative and non-increasing on $\left[0, v^{*}\right]$ (Lemma 1(ii)) and vanishes on $\left[v^{*}, 1\right]$. Therefore,

$$
\frac{\theta\left(v_{1}\right) \phi\left(v_{1}\right)}{v_{1}}-\frac{\theta\left(v_{2}\right) \phi\left(v_{2}\right)}{v_{2}} \geq 0
$$

in all cases. Now, if $u_{2} \leq v^{*}$, then $u \rightarrow \phi(u) / u$ is non-increasing on the considered interval and the conclusion follows. It $v^{*} \leq v_{1}$, then $\theta\left(v_{1}\right)=\theta\left(v_{2}\right)=0$ and the result is proved. 